Proc. Indian Acad. Sci., Vol. 87 A (E \& P Sciences), No. 3, March 1978, pp. 13-21, (C) printed in India.

\title{
Cavity radius estimation for contained peaceful nuclear explosions-An analytic approach
}

\author{
M P RANGA RAO* and S K SIKKA \\ Neutron Physics Section, Bhabha Atomic Research Centre, Bombay 400085 \\ *Mathematics Department, Indian Institute of Technology, Bombay 400076 \\ MS received 4 November 1977; revised 27 January 1978
}

\begin{abstract}
An analytical method based on Penny-Taylor model has been modified and applied for the estimation of the final cavity radius for contained peaceful nuclear explosions. The calculated cavity radii for some nuclear explosions in granite, alluvium and sandstone rocks are in good agreement with measured values.
\end{abstract}

Keywords. Peaceful nuclear explosions; contained explosions; nuclear cavity; analytical model.

\section{Introduction}

One of the important parameters in contained peaceful underground nuclear explosions is the final size of the explosion produced cavity, before complete or partial collapse of the overbearing rock takes place. Methods commonly employed to estimate this size use numerical rock mechanics computer codes (Cherry and Petersen 1970, Michaud and Maury 1970; Sikka et al 1975) or scaling laws (Nordyke 1970). In the computer methods, the medium, in which the explosion takes place, is divided into a number of meshes and the hydrodynamic equations expressed in finite difference form solved numerically for each mesh for time $(n+1) \Delta t$ from the knowledge of the stress field at time $n \Delta t$. The time step $\Delta t$, which is mesh size dependent can be as low as $\sim 10 \mu \mathrm{sec}$. Since the final size of the cavity is reached in many tens of milliseconds, the number of cycles of calculation is very large, thereby the numerical method requiring large computer time. The scaling laws, on the other hand are fast, but have limited applications as (i) the scaling constant has to be empirically determined from a number of nuclear detonations in a given rock medium and (ii) the form of the scaling law to be used may not be known exactly (see Nordyke 1970 for the difference between the USA and French forms).

The need for a rapid analytical type model was recognised quite early and a number of methods with varying degrees of approximation developed for cavities produced by chemical explosives (Hill 1950; Chadwick 1959; Chadwick et al 1964). However, these methods have not been thoroughly tested against experimental data and to the best of our knowledge, none of the methods has been modified and applied to the nuclear case. We modified the Penny-Taylor method as given by Chadwick et al (1964) and found that the calculated cavity radii for some nuclear detonations in granite, alluvium and sandstone rocks are in good agreement with measured or code calculated values. This paper describes the results of this investigation. 


\section{Penny-Taylor method}

In this model, as described and modified by Chadwick et al (1964), the earth medium is assumed to be an 'ideal soil' which is a solid that obeys Hooke's law within the elastic region and Coulomb's criterion at yield under the restriction of perfectly plastic flow and no density change. The pressure disturbance at any time, $t$, after the detonation is assumed to divide the medium into three zones (i) cavity region of radius $R$, with $R=R_{0}$ and $d R / d t=0$ at $t=0$, (ii) plastic region and (iii) elastic region. The equations of motion are then integrated in elastic and plastic regions separately. By matching the solutions at elastic-plastic and cavity-plastic region interfaces, the relation between $P(R)$, the cavity pressure and the cavity radius $R$ is of the form (eq. 6.23 of Chadwick et al 1964).

$$
K_{1} x \ddot{x}+2\left(K_{1}-K_{2}\right) \dot{x}^{2}+K_{3}-\theta(x)=0
$$

where $x\left(=R / R_{0}\right)$ is the dimensionless cavity radius, $\dot{x}$ and $\ddot{x}$ are the derivatives w.r.t. time $t$, the constants $K_{1}, K_{2}$ and $K_{3}$ are

$$
\begin{aligned}
& K_{1}=\frac{1+a}{1-a}\left\{1-n^{-(1-\alpha) /(1+a)}\right\}, \\
& K_{2}=\frac{1+a}{2(2+a)}\left\{1-n^{-2(2+a) /(1+a)}\right\}, \\
& K_{3}=1 / E(Y / a+\pi)\left\{\frac{3(1+a)}{3+a} n^{2 \alpha /(1+a)}-1\right\} \text { and } \\
& \theta(x)=(P(R)-\pi) / E \\
& a=2 \sin \phi /(1-\sin \phi), \\
& Y=2 c \cos \phi /(1-\sin \phi), \\
& n^{3}=\frac{2 \mu(3+a)}{3(Y+a \pi)}
\end{aligned}
$$

$E=$ Young's modulus, $\mu=$ shear modulus and $\pi=\rho g h$, the overburden pressure for depth of emplacement of the explosive charge, $h . c$ and $\phi$ are constants in the Coulomb's law for yield

$$
T_{s}=c-\sigma_{n} \tan \phi
$$

Here $\sigma_{n}$ and $\mathfrak{T}_{s}$ are the normal and shear stresses exerted across a plane surface element of a typical point of a portion of soil.

Equation (1) is a non-linear second order differential equation which can be integrated for $R$ as a function of time, if $P(R)$ and other initial conditions are specified. 


\section{Application to nuclear explosions in rocks}

The two main assumptions of the model described above are:

(i) incompressibility of the earth medium, and

(ii) the application of the Coulomb's law for the onset of plastic flow.

The rocks on the other hand are compressible and fail through brittle fracture. To examine the effect of compressibility on the size of the cavity, we analysed the nuclear cavity growth curves ( $R$ vs $t$ ) generated from computer codes and found that the final stages of their growth (see figure 1 for one such cavity) could be very well approximated by Sedov's (1959) formula for an incompressible fluid. This means that compressibility of the rocks should have very small influence on the final cavity radius. Chadwick (1959) has also observed that the neglect of compressibility has only little effect on the numerical values of the cavity radii.

Mogi (1974) has examined the question of the applicability of the Coulomb's criterion for rocks and shown that the law is approximately valid for the description of the pressure dependence of the fracture except for very low pressures and near the brittle-ductile transition point. Also for rocks, the yield point does not differ very much from the fracture point. In view of the above, one can continue to use this law provided the constants $c$ and $\phi$ in eq. (2) are determined from the experimental (e.g. triaxial) fracture stress data.

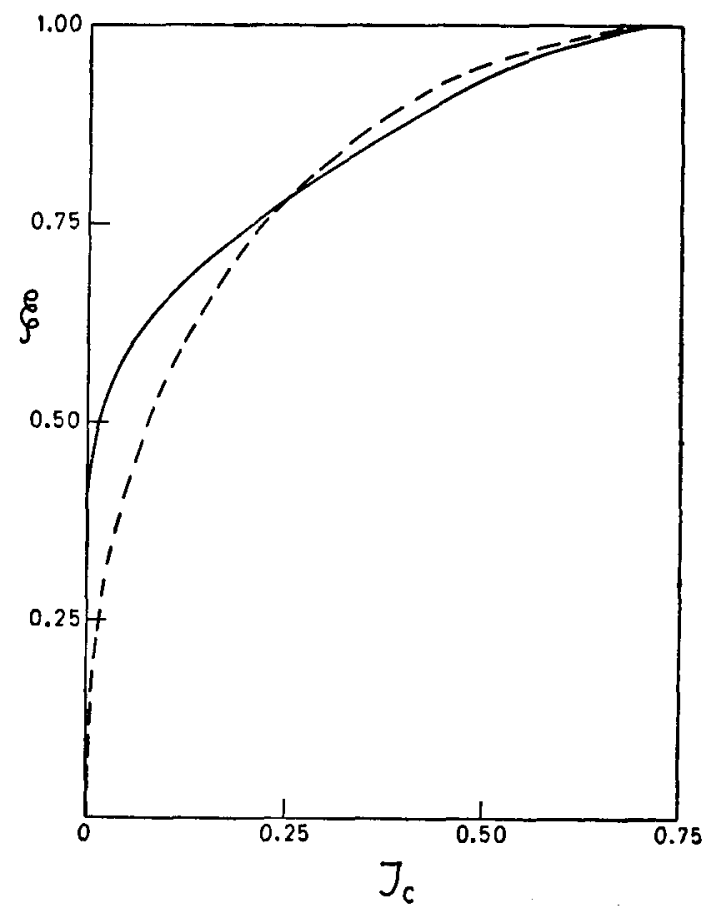

Figure 1. Expansion law for the cavity. Sedov's solution ---, $\xi=R / R_{\max }, R_{\max }$ $=\left(\frac{3}{25 \pi} \frac{W}{P_{1}}\right)^{1 / 3}, \mathcal{F}_{c}=\frac{t(25 \pi)^{1 / 3} \sqrt{2} P_{1}^{5 / 6}}{3^{5 / 6} \rho^{1 / 2} W^{1 / 3}}$ and $P_{1}$ the overburden pressure. Code calculated curve $\longrightarrow$ (case 4 of Terhune, 1971). 
As pointed out in section $2, R$ as a function of time can be calculated from eq. (1) provided we specify $P(R)$. Now $P(R)$ curves are easily obtained from nuclear code calculations and many authors have given them in their reports. The form of these curves suggest that these can be fitted to the following expression*

$$
\begin{aligned}
P(x) & =P_{0} x^{-3 \gamma_{1}}\left(1 \leqslant x \leqslant x_{1}\right) \\
& =P_{0} x_{1}^{3\left(\gamma_{2}-\gamma_{1}\right)} x^{-3 \gamma_{2}}\left(x_{1} \leqslant x \leqslant x_{2}\right) \\
& =P_{0} x_{1}^{3\left(\gamma_{2}-\gamma_{1}\right)} x_{2}^{3\left(\gamma_{3}-\gamma_{2}\right)} x^{-3 \gamma_{3}}\left(x>x_{2}\right) .
\end{aligned}
$$

Here $x$ is again the dimensionless cavity radius, $R / R_{0}$. For $P_{0}$ and $R_{0}$, we have adopted the Bubble model of Butkovich (1967), in which $P_{0}$ is given by the pressure at the completion of the vaporization phase of the rock and $R_{0}=R_{1} W^{1 / 3}$ (W= yield of the nuclear explosive in kilotons of TNT). $R_{1} \simeq 2$ meters for most cases. The exact value, however, can be evaluated from the $\left(R_{1}\right.$ vs $\left.E_{0}\right)$ curve given by Butkovich (1967).

For numerical work, we expressed the solution of eq. (1) in the following form:

Region $1 \leqslant x \leqslant x_{1}$

$$
\begin{aligned}
& T=\int_{1}^{x} \frac{d x}{\sqrt{f_{1}(x)}} \\
& f_{1}(x)=\frac{2}{K_{1}}\left[\frac{P_{0} x^{-3 \gamma_{1}}}{E\left(c_{1}-3 \gamma_{1}\right)}-c_{2}\right]+c_{3} x^{-c_{1}} \\
& c_{1}=4\left(1-K_{2} / K_{1}\right) \\
& c_{2}=\left(\pi / E+K_{3}\right) / c_{1} \\
& c_{3}=\frac{\left(\frac{P_{0}-\pi}{E}-K_{3}\right)}{2\left(K_{1}-K_{2}\right)}-\frac{2}{K_{1}}\left[\frac{P_{0}}{E\left(c_{1}-3 \gamma_{1}\right)}-c_{2}\right]
\end{aligned}
$$

Region $x_{1} \leqslant x \leqslant x_{2}$

$$
\begin{aligned}
& T-T_{1}=\int_{x_{1}}^{x} \frac{d x}{\sqrt{f_{2}(x)}} \\
& f_{2}(x)=\frac{2}{K_{1}}\left[\frac{P_{0} x^{3\left(y_{2}-\gamma_{1}\right)} x^{-3 \gamma_{2}}}{E\left(c_{1}-3 \gamma_{2}\right)}-c_{2}\right]+c_{4} x^{-c_{1}}
\end{aligned}
$$

*This is a generalization of the two $\gamma$-expression (4-1) of Chadwick et al (1964). For most nuclear acses this three $\gamma$ expression is adequate. 


$$
\begin{aligned}
& c_{4}=x_{1}^{c_{1}}\left[f_{1}\left(x_{1}\right)-\frac{2}{K_{1}}\left\{\frac{P_{0} x_{1}^{-3 y_{1}}}{E\left(c_{1}-3 \gamma_{2}\right)}-c_{8}\right\}\right] \\
& T_{1}=\int_{1}^{x_{1}} \frac{d x}{\sqrt{f_{1}(x)}}
\end{aligned}
$$

Region $x>x_{2}$

$$
\begin{aligned}
& T-T_{2}=\int_{x_{2}}^{x} \frac{d x}{\sqrt{f_{3}(x)}} \\
& f_{3}(x)=\frac{2}{K_{1}}\left[\frac{P_{0} x_{1}^{3\left(y_{2}-y_{1}\right)} x_{2}^{3\left(y_{3}-y_{3}\right)} x^{-3 y_{2}}}{E\left(c_{1}-3 \gamma_{3}\right)}-c_{2}\right]+c_{5} x^{-c_{1}} \\
& c_{5}=x_{2}^{c_{1}}\left[f_{2}\left(x_{2}\right)-\frac{2}{K_{1}}\left\{\frac{P_{0} x_{1}^{3\left(y_{2}-\gamma_{1}\right)} x_{2}^{-3 \gamma_{2}}}{E\left(c_{1}-3 \gamma_{3}\right)}-c_{2}\right\}\right] \\
& T_{2}=T_{1}+\int_{x_{1}}^{x_{1}} \frac{d x}{\sqrt{f_{2}(x)}}
\end{aligned}
$$

Here $T$ is the non-dimensionless time $=c_{0} t / R_{0}$ and $c_{0}^{8}=E / \rho$.

We have calculated nuclear explosions in three rocks, granite, alluvium and sandstone. The values of the parameters required in the above expressions are derived from published data and are listed in table 1. The various integrals were evaluated by simple quadrature. Each cavity radius calculation took about $15 \mathrm{sec}$ of computer time on the BESM-6 machine at Trombay. The calculated values of the $R_{\max }$ are given in table 2, alongwith the measured ones. Also in figure 2, we have compared the experimental values of $R_{\max }$ for 30 tests in alluvium* conducted in USA (all scaled to a common yield of $1 \mathrm{kt}, R_{\max }^{\mathrm{S}}=R_{\max } / W^{1 / 3}$ ) with our calculated curve as a function of the depth of the burst.

\section{Discussion}

As can be seen, the agreement between our calculated values and measured ones is satisfactory. The deviations are within the uncertainties of experimental values. This justifies the assumptions regarding the incompressibility and description of the failure. The dependence of $R_{\max }$ on the depth of emplacement (figure 2) is very well reproduced. This is heartening, since these 30 USA tests have been carried out in alluvium with different medium properties and only average values of parameters

"Data taken from Higgins and Butkovich (1967).

P. (A) -2 


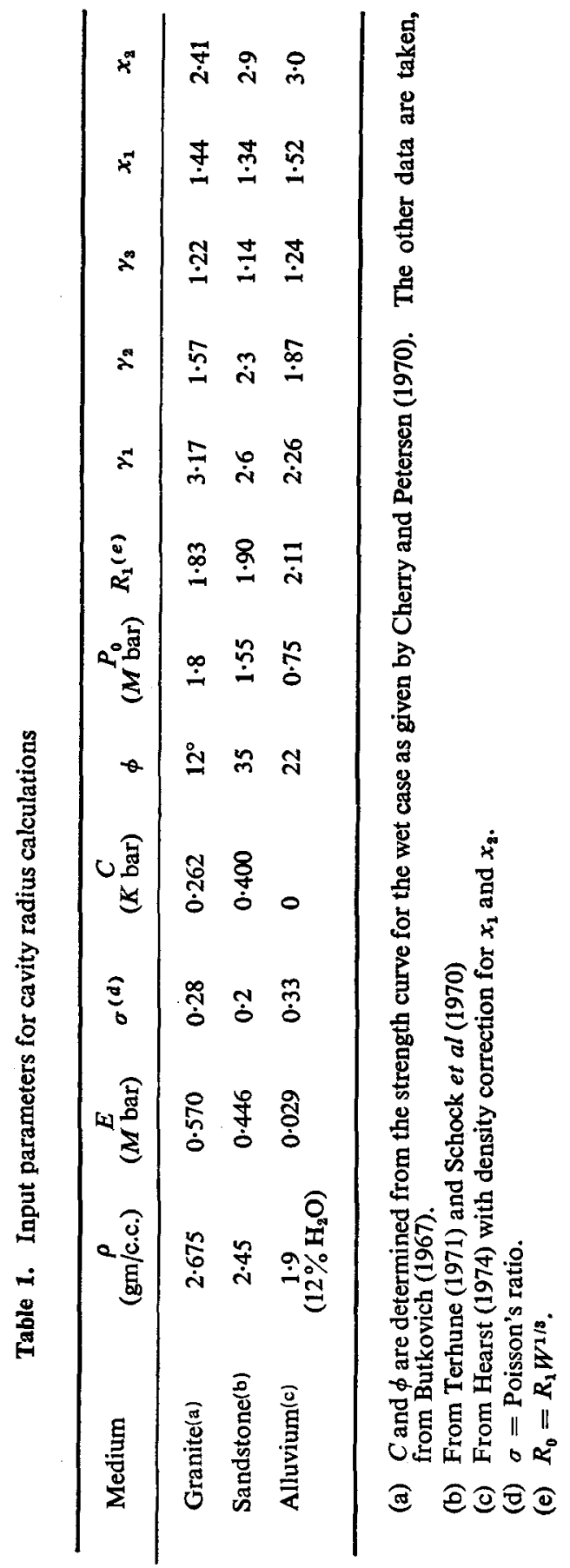


Table 2. Numerical values for cavity radii

\begin{tabular}{|c|c|c|c|c|c|c|}
\hline Event & Medium & Yield & Depth & $\begin{array}{c}R_{\max } \\
\text { (present } \\
\text { study) }\end{array}$ & $\underset{\text { (measured) }}{R_{\mathrm{max}}}$ & $\begin{array}{l}R_{\max } \\
\text { (SOC) }\end{array}$ \\
\hline Hardhat & Granite & $4.8 \mathrm{kt}$ & $286 \mathrm{~m}$ & $21.1 \mathrm{~m}$ & $20.2 \mathrm{~m}$ & $20.4 \mathrm{~m}^{*}$ \\
\hline Shoal & Granite & $12 \cdot 8$ & 367 & 28.9 & $27 \cdot 1$ & - \\
\hline Pile-driver & $\begin{array}{l}\text { Granite } \\
\text { Sandstone } \\
\text { Sandstone } \\
\text { Sandstone }\end{array}$ & $\begin{array}{l}56 \\
1 \\
20 \\
40\end{array}$ & $\begin{array}{l}463 \\
270 \\
2700 \\
2700\end{array}$ & $\begin{array}{c}47 \cdot 2 \\
7 \cdot 6 \\
19 \cdot 1 \\
24 \cdot 0\end{array}$ & $\frac{44 \cdot 5}{=}$ & $\begin{array}{l}7 \overline{2 * *} \\
15 \cdot 3 \\
19 \cdot 5\end{array}$ \\
\hline Hupmobile & Alluvium & $7 \cdot 4$ & 246 & 32.6 & 32 & - \\
\hline
\end{tabular}

*From Cherry and Petersen (1970)

**From Terhune (1971)

(as quoted in table 1) are used in our calculations. The effect of the strength of the medium is automatically included in the final formulae for $R(t)$. The effect of the depth of burst and the strength of the rock on $R_{\max }$ becomes more transparent from the following analysis of eq. (6). Using the fact that when $R=R_{\max }\left(x=x_{\max }\right.$ ), $\dot{x}=0$, on can write from (6)

$$
\frac{2}{K_{1}}\left[\frac{P_{0} x_{1}^{3\left(\gamma_{2}-\gamma_{1}\right)} x_{2}^{3\left(\gamma_{3}-\gamma_{3}\right)} x_{\max }^{-3 \gamma_{3}}}{E\left(c_{1}-3 \gamma_{3}\right)}-c_{2}\right]+c_{5} x_{\max }^{-c_{1}}=0 .
$$

Now the value of $c_{1}$ is nearly equal to 4 and $x_{\max }$ for nuclear explosions is $>2.5$ (see table 1). This makes the second term in (7) small. Omitting this term in (7) and after some rearrangement we recover a scaling law for $R_{\max }$ for yield $W$ of the nuclear explosive as

$$
R_{\max } \simeq R_{1} W^{1 / 3}\left[\frac{c_{1} P_{0} x_{1}^{3\left(\gamma_{2}-\gamma_{1}\right)} x_{2}^{3\left(\gamma_{3}-\gamma_{2}\right)}}{\left(c_{1}-3 \gamma_{3}\right)\left(\pi+E K_{3}\right)}\right]^{1 / 3 \gamma_{3}}
$$

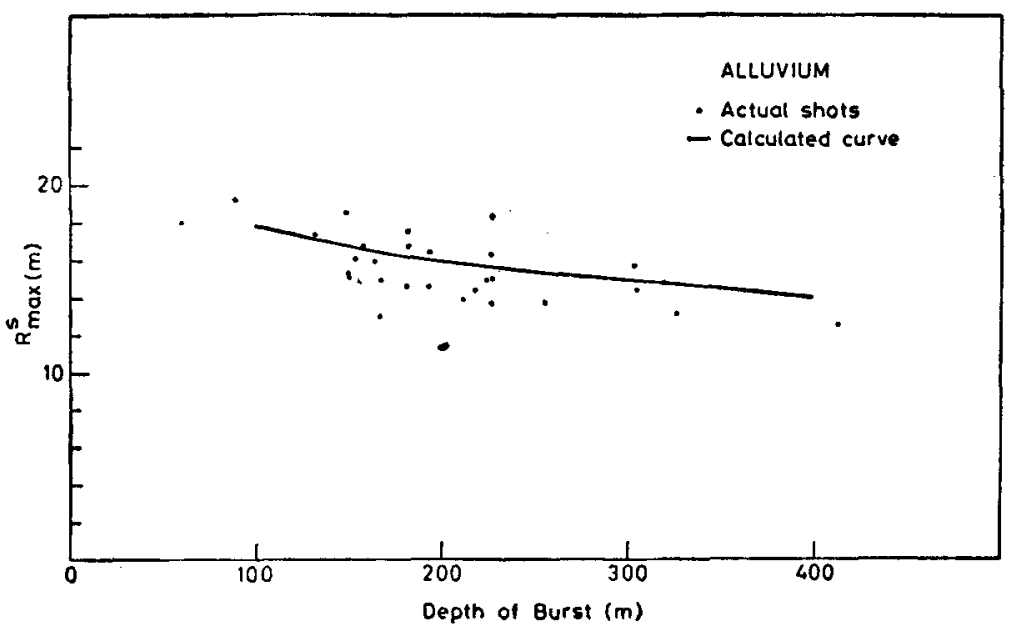

Figure 2. Comparison of calculated and measured values of $R_{\max }^{S}\left(=R_{\max } / W^{2} n\right.$ as a function of depth of burst for nuclear explosions in alluvium medium. 
Here

$$
\pi+E K_{3}=\pi+(c \cot \phi+\pi)\left[\frac{3(1+a)}{(3+a)}\left\{\frac{2 \mu[(3 / a)+1]}{3(c \cot \phi+\pi)}\right\}^{2 a / 3(1+a)}-1\right],
$$

with $\pi=\rho g h$ and $c_{1}$ and $a$ related to $\phi$ as given in eq. (1). Comparing this with standard form for the cavity radius scaling law (Michaud 1970).

$$
R_{\max }=\frac{P_{0}^{1 / 8^{y}} R_{1} W^{1 / 8}}{P_{c}^{1 / 8^{7}}}
$$

one gets, $P_{c}$, the cavity pressure at equilibrium as

$$
P_{c}=\left(c_{1}-3 \gamma_{3}\right)\left(\pi+E K_{\mathrm{a}}\right) / c_{1} .
$$

This coupled with eq. (8) shows that the dependence of $R_{\max }$ on $\pi$ and strength properties is intermingled and is more complicated than the simple form, assumed in scaling laws

$$
P_{c}=\pi+c_{s}
$$

Here $c_{s}$ is the effective shear strength of the rock. Our eq. (10) has the advantage over (11) in the sense that it can be evaluated from medium properties, while for $c_{s}$, one does not a priori, know its relationship to say, the strength measured in laboratory on competent rock samples. For wagon-wheel sandstone, code calculations (Terhune 1971) have shown that $c_{s}=c$ (see eq. (2)), while Allen and Duff (1969) have shown for tuff that it should be much less than $c$. Further, it can be seen from eq. (8), that the strength curve as a whole (i.e. both $c$ and $\phi$ in our case) affects $R_{\max }$, unlike that in eq. (11). For sandstone rock ( $1 \mathrm{kt}, h=270 \mathrm{~m}$, case given in table 2), $R_{\max }$ changed from $7.6 \mathrm{~m}$ to $6.7 \mathrm{~m}$ as $\phi$ was changed from $35^{\circ}$ to $48^{\circ}$ [the latter being the average value of $\phi$ for sandstone, (Mogi 1974)]. The rate of cavity growth was also lower.

\section{Conclusions}

We have modified and applied the Penny-Taylor model for the calculation of the radius of nuclear explosion produced cavities. The numerical form of the cavity growth eqs [(4), (5) and (6)] presented is easier to handle than the original equation of Chadwick et al (1964). The method is simple to use as it requires few inputs about the material properties of the surrounding rock than rock mechanics codes and is fast. The results obtained show that the cavity size is not very critically dependent upon the compressibility of the medium. However, the radius is found to be dependent upon the full strength curve of the rock.

\section{Acknowledgement}

We thank Dr R Chidambaram for suggesting the problem and for many discussions. 


\section{References}

Allen R T and Duff R E 1969 Nucl. Appl. 6567

Butkovich T R 1967 Rep. UCRL-14729

Chadwick P 1959 J. Mech. Appl. Math. 1252

Chadwick P, Cox A D and Hopkins H G 1964 Philos. Trans. R. Soc. A256 235

Cherry J T and Petersen F L 1970 Proc. IAEA Peaceful Nucl. Explosions Conf. 1241

Hearst J R 1974 Rep UCID-16470

Higgins G H and Butkovich T R 1967 Rep. UCRL-50203

Hill R 1950 The Mathematical Theory of Plasticity (Oxford: Clarendon Press) p. 104

Michaud L 1970 Rep CSA-R 3594

Michaud L and Maury J 1970 Proc. IAEA Peaceful Nucl. Explosions Conf. 1139

Mogi K 1974 Tectonophysics 21273

Nordyke M D 1970 Proc. IAEA Peaceful Nucl. Explosions Conf. 149

Schock R, Stephens D and Heard H 1970 Rep. UCRL - 50963

Sedov L I 1959 Similarity and Dimensional Methods in mechanics (New York: Academic Press)

Sikka S K, Gupta S C and Chidambaram R 1975 Ann. Rep. of Neutron Physics Section, BARC Terhune R W 1971 Rep. UCRL - 50993 Rev. 1. 